\title{
STATUS EM SOCIEDADES PÓS-MODERNAS: A RENOVAÇÃO DE UM CONCEITO*
}

\section{Michèle Ollivier ${ }^{* \star}$}

\begin{abstract}
"Tomemos, como exemplo sumário, o bibliotecário de uma universidade ou um alto funcionário administrativo, tal como o tesoureiro ou chefe de secretaria. Seu status difere daquele de um professor porque seu papel e as funções de seu cargo são diferentes das de um professor. Mas seu escalonamento no quadro da universidade pode ser o mesmo. Pode, entretanto, ter um status social mais baixo na comunidade em geral do que aquele da maioria dos professores (talvez devido às origens de sua família), e uma avaliação um tanto baixa no que toca à inteligência, cultura geral ou facilidade no trato social. Em consequência de todos esses fatores sua posição na universidade não é aquela que ele gostaria que fosse" Marshall (1964, p. 229) ${ }^{1}$
\end{abstract}

\footnotetext{
* Agradeço a ajuda e os comentários de Aage Sørensen, Terry Nosanchuck e François Gobeil. Esta pesquisa foi financiada parcialmente por uma bolsa de pós-doutorado fornecida pelo Canadian Social Sciences and Humanities Research Council e por recursos concedidos pela Carleton University.

** Tradução de Eugênio Braga.

${ }^{1}$ N.T.: Mantive, para este extrato em epígrafe - de um texto escrito por T. H. Marshall originalmente em 1954 [A note on 'status'] - a tradução publicada em português, realizada por Meton Porto Gadelha [Uma nota sobre status, pp. 172-180], disponível no livro Cidadania, classe social e status (Rio de Janeiro: Zahar Editores, 1967), versão do original em inglês de 1963, Sociology at the crossroads and other essays (London: Heinemann Educational Books Ltd.), fonte também da edição utilizada pela autora, de 1964, com outro título. O leitor pode ter uma pequena amostra das questões de tradução comparando-o com o extrato original: "Let us take, as a summary example, a University Librarian or senior administrative officer such as Registrar or Bursar. His status differs from that of a Professor because his role and the functions of his post differ from those of a Professor. But his university rank may be the same. He may, however, have a lower social status in the community at large than most Professors (perhaps because of his family origins), and a rather low rating for intelligence or general culture or social graces. In consequence of all these factors his standing in the university is not quite what he would like it to be".
} 
Status é um conceito com vários significados, e muitos destes envolvem uma combinação de dois elementos bem diferentes. No primeiro, status se refere à estima ou desprezo, deferência ou depreciação concedida a indivíduos ou grupos percebidos como superiores ou inferiores. Nesse sentido, se refere às avaliações positivas e negativas feitas pelas pessoas sobre as outras e é sinônimo de prestígio. Como esse primeiro sentido para status diz respeito a um sentimento na mente das pessoas, remete a um aspecto especificamente simbólico da desigualdade. No segundo, status pode se referir também a posições na estrutura social, de um modo completamente independente de avaliações individuais de superioridade e inferioridade. Tal como nas escalas "puras" de status socioeconômico, por exemplo, construídas combinando uma série de atributos das ocupações (Nam e Terrie, 1982; Powers, 1982). Aqui, status se refere simplesmente a uma gradação de posições definidas

42 a partir de recursos socioeconômicos. Da mesma forma, conceitos que indicam status jurídicos, como os de estado civil [marital status], dizem respeito a direitos e deveres de categorias sociais também de uma maneira que tem pouca relação com status no sentido de prestígio.

Mesmo sendo conceitos tão distintos, status como avaliações de superioridade e inferioridade e status como posições sociais estiveram frequentemente ligados na sociologia clássica. Weber, por exemplo, considerava que o status, na qualidade de reivindicações [ claims] por estima social, é um meio através do qual grupos dentro de uma comunidade legitimam seus privilégios legais ou costumeiros. Em Durkheim, avaliações coletivas acerca do valor social das posições determinam a distribuição desigual das recompensas materiais e formam a espinha dorsal dos sistemas de estratificação. Tomado a partir de seu sentido sociológico quintessencial, status se refere às avaliações coletivas de superioridade e inferioridade que adquirem uma existência 
além das crenças individuais e que influenciam as relações sociais de várias maneiras.

Quer indicando posições sociais per se ou avaliações sociais, os conceitos de status também se diferenciam de acordo com o modo como representam os pontos da estrutura social. Essas representações podem ser classificadas em três categorias principais. Na primeira, status se refere a um ordenamento de unidades, posições sociais, na maioria das vezes relacionadas a características selecionadas. Escalas de prestígio ocupacional e de status socioeconômico, por exemplo, são gradações sintéticas de posições sociais. ${ }^{2}$ Estas escalas são construídas de maneiras bastante diferenciadas, e isso leva a uma variedade de interpretações. As medidas "puras" de status socioeconômico são normalmente construídas combinando vários atributos das ocupações. São classificações nominais das posições ao longo de algumas dimensões da desigualdade e são compatíveis com um conjunto de perspectivas teóricas. As escalas de prestígio ocupacional, pelo contrário, se baseiam em avaliações populares sobre as posições sociais e ainda há divergência sobre o que exatamente elas medem. Autores as interpretam tanto como medidas de posição social quanto como avaliações do valor das ocupações. Quando interpretadas seguindo esta última leitura, ou seja, como avaliações normativas, normalmente estão inseridas dentro de teorias de estratificação neodurkheimianas, enfatizando a ordem e o consenso.

Na segunda categoria, status é conceitualizado como um atributo de grupos cujas reivindicações por estima social os autorizam a direitos e deveres específicos. O tratamento mais influente na sociologia nesse sentido está na obra de

\footnotetext{
${ }^{2}$ O termo "gradação sintética" foi cunhado por Ossowski (1963), dentro de sua proposta que considera as gradações como um entre três tipos de representações da estrutura social. Os demais tipos são esquemas categóricos. A classificação apresentada neste texto foi adaptada de modo a incluir um terceiro tipo de representação.
} 
Weber (1978), em que o status é um atributo dos grupos que utilizam sua superioridade para, legitimamente, fechar oportunidades a não membros. Outro exemplo é o conceito de cidadania de Marshall (1964), que é um status no sentido jurídico. Cidadania não implica estilos de vida específicos nem é legitimada por meio de ideias de superioridade e inferioridade coletivas, mas define os direitos e deveres dos cidadãos dentro de um Estado-nação e exclui não membros da participação política, econômica e social. Neste sentido categórico, o status é normalmente mais do que uma gradação descritiva porque implica alguma concepção dos mecanismos que criam e sustentam a desigualdade. Em oposição às gradações, os conceitos categóricos estão, na maioria das vezes, associados a teorias que enfatizam o conflito e a dominação. Ao invés de refletir os valores e interesses de toda uma comunidade, o status é percebido aqui como a serviço dos interesses de grupos específicos.

44 Finalmente, em comunidades frouxamente estruturadas ou em pequenos grupos, status pode ser considerado como um atributo dos indivíduos. Refere-se, dessa forma, ao respeito obtido por indivíduos que mostram qualidades consideradas excepcionais ou que fornecem serviços que não podem ser trocados reciprocamente (Homans, 1950; Blau, 1967; Bourdieu, 1977). Status com este sentido está geralmente associado com análises de relações interpessoais a nível micro ou com contextos sociais pouco diferenciados [low-grid], isto é, aqueles nos quais existem poucas classificações institucionais e poucas restrições para as relações entre os indivíduos (Douglas, 1979). Como atributo individual, o status pode estar relacionado com teorias de estratificação tanto centradas na ordem quanto no conflito. Assim, pode ser visto como uma recompensa por qualidades e realizações excepcionais, consensualmente avaliadas e que beneficiam todo o grupo (Homans, 1950; Blau, 1967) ou como uma dentre as variadas formas com as quais 
indivíduos e grupos exercem poder uns sobre os outros (Bourdieu, 1977; Blau, 1967).

A Tabela 1 resume estes conceitos e medidas de status. No topo, estão os conceitos que se referem ao status como um atributo dos indivíduos. Quando conceitualizado dessa forma, o status é por definição um conceito avaliativo, porque considera os indivíduos e não as posições que ocupam na estrutura social. Na parte intermediária da tabela, estão os conceitos gradativos. A coluna à esquerda inclui gradações estritamente posicionais, tais como as escalas de status socioeconômico, que não se referem a avaliações de superioridade e inferioridade. Na coluna à direita estão incluídas as gradações normativas, associadas com as teorias neodurkheimianas e funcionalistas normativas que consideram o status como uma classificação moral. Na base da tabela estão os conceitos categóricos. Estes incluem os conceitos normativos inspirados a partir dos trabalhos de Weber sobre os grupos de status (Bourdieu, 1979; Collins, 1979; Murphy, 1988) e os conceitos posicionais, tais como o de status jurídico de Marshall e os intitulamentos [entitlements] políticolegais de Turner (1988).

O principal objetivo deste artigo é o de avaliar a relevância dos conceitos categóricos e gradativos de status herdados de Durkheim e Weber para a compreensão do papel, se é que esse papel existe, das avaliações de superioridade e inferioridade na produção da desigualdade nas sociedades contemporâneas. Eu defendo que os conceitos clássicos de status têm sérias limitações. As gradações normativas funcionalistas indicam que as avaliações coletivas são os elementos centrais de todos os sistemas de estratificação. No entanto, o pressuposto de uma ordem de status única e consensualmente avaliada como essencial para a integração social é extremamente problemático nas sociedades caracterizadas por filiações múltiplas e relacionamentos indiretos. As gradações posicionais, desenvolvidas como uma extensão 
do funcionalismo normativo, são indicadores úteis da posição social relacionada com recursos socioeconômicos, mas não mensuram status no sentido simbólico. O conceito categórico de status de Weber aponta para a utilização das avaliações coletivas pelos grupos de modo a legitimar a exclusão legal ou costumeira, mas não é muito útil para compreender como as avaliações coletivas são utilizadas para criar ou sustentar a desigualdade entre indivíduos formalmente iguais competindo em mercados abertos.

\section{Tabela 1}

Conceitos e medidas de status

\begin{tabular}{|c|c|c|}
\hline STATUS & Posicional & Avaliativo/Normativo \\
\hline Individual & & $\begin{array}{c}\text { Homans }(1950) \\
\text { Blau }(1967,1971)\end{array}$ \\
\hline Gradativo & $\begin{array}{c}\text { Sorokin (1964) } \\
\text { Lenski (1984) } \\
\text { Medidas de status socioeconômico } \\
\text { baseadas em escalas de prestígio } \\
\text { (Blishen e Carroll, 1982; Duncan, 1961) } \\
\text { Medidas puras de status } \\
\text { socioeconômico (Nam e Terrie, 1982) }\end{array}$ & $\begin{array}{c}\text { Durkheim (1960a) } \\
\text { Davis e Moore (1945) } \\
\text { Parsons (1954) } \\
\text { Warner et alii (1960) } \\
\text { Shils (1975) } \\
\text { Escalas de prestígio ocupaciona } \\
\text { (Reiss, 1961; Treiman, 1977) }\end{array}$ \\
\hline Categórico & $\begin{array}{l}\text { Marshall (1964) } \\
\text { Halsey (1986) } \\
\text { Turner (1988) }\end{array}$ & $\begin{array}{c}\text { Weber }(1978) \\
\text { Bourdieu }(1977,1979) \\
\text { Collins }(1979) \\
\text { Murphy }(1988)\end{array}$ \\
\hline
\end{tabular}

\section{Gradações normativas}

O conceito de status como uma hierarquia de posições ancorada em um sistema de valores compartilhados dominou os estudos de estratificação das décadas de 1940 a 1960. Suas raízes remontam ao interesse de Durkheim pela coesão social e, mais precisamente, à sua visão de que as avaliações compartilhadas das posições sociais são essenciais para a estabilidade e para a integração:

"Com efeito, em todo momento da história existe um sentimento obscuro, na consciência moral das sociedades, 
acerca do valor respectivo aos diferentes serviços sociais, da remuneração merecida por cada um e, consequentemente, do grau de conforto apropriado, em média, aos trabalhadores de cada ocupação. As diferentes funções são hierarquizadas pelas opiniões e um certo coeficiente de bem-estar é atribuído a cada uma, de acordo com a posição que ocupa na hierarquia” (Durkheim, 1960a, pp. 275-276).

Para Durkheim, essas avaliações compartilhadas das posições formam uma classificação moral cujo poder transcende os interesses privados porque emana da própria sociedade. Classificações morais estabelecem os limites para os interesses e apetites individuais ao legitimar a alocação desigual das recompensas e o posicionamento dos indivíduos no interior da hierarquia de posições. Quando essas avaliações não existem ou estão enfraquecidas como resultado de crises sociais, as paixões não encontram obstáculos e a sociedade entra em um Estado de desregulação, ou anomia (Durkheim, 1960a).

O interesse de Durkheim pela classificação ocupacional não desempenha um papel central em sua sociologia, mas forma a espinha dorsal dos conceitos funcionalistas normativos de status. Parsons, que articulou os principais aspectos dessa perspectiva no contexto de sua teoria da ação, partilhou da ideia durkheimiana de que um sistema de valores coletivos é uma condição para a estabilidade dos sistemas sociais. Parsons usa o termo status como equivalente de posição e o de estratificação para se referir ao ordenamento concreto das posições de acordo com um sistema comum de valores. Assim, Parsons definiu o status em termos estritamente posicionais, ou seja, como pontos na estrutura social, mas deixou claro que o status implica necessariamente alguma forma de avaliação. Como a ação social é orientada para objetivos, Parsons sustentou que todos os componentes dos sistemas de ação são sujeitos a processos avaliativos. São essas avaliações dos diferentes status que dão origem à estratificação: "Estrati- 
ficação, no seu aspecto valorativo, é portanto o ordenamento das unidades em um sistema social de acordo com os padrões do sistema comum de valores" (Parsons, 1954, p. 388)

Desse modo, as hierarquias de status não são o resultado de lutas pelo poder entre grupos, mas uma característica necessária de todas as sociedades. As posições mais elevadas em um sistema de status são aquelas mais valorizadas por uma coletividade, em razão de serem funcionalmente essenciais para a sobrevivência da sociedade (Barber, 1957; Davis e Moore, 1945; Warner et al., 1960), de sua proximidade dos valores e instituições centrais que fornecem significado e ordem para a vida humana (Shils, 1975) ou porque exigem habilidades raras (Davis e Moore, 1945).

De acordo com o funcionalismo normativo, o sistema de status beneficia toda a sociedade ao realizar duas importantes funções. Seguindo Durkheim, as hierarquias de status são essenciais para a integração social. Elas garantem que as 48 recompensas vinculadas às posições sociais correspondam às utilidades sociais e, portanto, que as desigualdades sociais sejam percebidas como necessárias e legítimas:

"Em outras palavras, na medida em que o sistema de estratificação é uma expressão ou resultado de julgamentos ordenadores diferenciais em relação a algum conjunto comum de valores, serve para integrar a sociedade. Os homens têm a sensação de que a justiça está sendo feita, e a virtude recompensada, quando são ordenados de modo claro como superiores e inferiores pelos valores-padrão de sua própria comunidade moral" (Barber, 1957, p. 7).

A segunda função é motivacional. Avaliações diferenciais resultam em recompensas diferenciais e essas, por sua vez, fornecem os incentivos para o recrutamento das pessoas mais competentes para as posições mais exigentes. Dessa maneira, a estratificação proporciona 
"por sua estrutura de ordenações diferenciais, um conjunto de facilidades e recompensas relativas para que as atividades valorizadas socialmente sejam realizadas e um conjunto de privações e punições relativas caso não sejam, ou não sejam muito bem, realizadas" (Barber, 1957, p. 7).

Isso não quer dizer que as posições são altamente valorizadas porque recebem altas recompensas. Pelo contrário, a alta recompensa é um meio de atrair candidatos qualificados para aquelas posições que já são altamente valorizadas.

Este conceito de status como hierarquia de posições baseada em valores compartilhados, com sua ênfase no papel das avaliações normativas na integração social, manteve-se como paradigma dominante nos estudos empíricos norte-americanos de estratificação dos anos 1940 aos 1960. Entre outras coisas, forneceu um enquadramento teórico para os estudos que pretendiam mapear a estrutura de status de comunidades inteiras. O objetivo era identificar grupos reais localizados nos vários níveis de status, tomando como base a observação da interação e as avaliações da comunidade. Muito embora o termo classe tenha sido muitas vezes preferido ao termo status, os dois conceitos estavam intimamente associados. Status era utilizado para indicar a hierarquia de valores subjacente, enquanto classe se referia a aglomerados de posições similares na hierarquia de status: "Classe designa um agregado de pessoas, dentro de uma sociedade, que possuem aproximadamente o mesmo status" (Shils, 1975, p. 249; ver também Barber, 1957, p. 73; Coleman e Rainwater, 1978, p. 17; Warner et al., 1960).

No entanto, com o enfraquecimento do funcionalismo e com a mudança do cenário das pesquisas de pequenas comunidades para grandes cidades, os conceitos normativos de status recebiam cada vez mais críticas. No nível teórico, as teorias neodurkheimianas e funcionalistas eram 
criticadas pela quase exclusiva ênfase nos aspectos normativos e integradores da estratificação e pela falta de interesse no poder, no conflito e na coerção. Seguindo Durkheim, o funcionalismo normativo prestou pouca atenção aos mecanismos e tipos de arranjos socioeconômicos responsáveis pela geração das desigualdades de riqueza e poder. A fusão de status e classe dificultou bastante a investigação da relação entre status como posições na estrutura social e status como avaliações coletivas de superioridade e inferioridade. As hierarquias de status eram percebidas como uma característica natural e necessária de todas as sociedades e, portanto, não eram consideradas problemáticas. A questão estava, antes, em garantir que os indivíduos pudessem se mover dentro da ordem de status de acordo com seus níveis de habilidade e esforço (Durkheim, 1960b; Warner et al., 1960).

No nível empírico, os sociólogos interessados em estu50 dar o status em grandes áreas metropolitanas se depararam com dificuldades metodológicas. Embora os métodos etnográficos possibilitassem retratos detalhados dos sistemas de status em pequenas comunidades, eles eram menos úteis para o estudo de grandes metrópoles, caracterizadas pelo anonimato, altos níveis de mobilidade geográfica e heterogeneidade ocupacional dentro da uma mesma área residencial. Para ultrapassar essas limitações, Warner e seus associados desenvolveram um "índice de características de status" baseado em atributos considerados altamente correlacionados com classe social, tais como ocupação, fonte de renda, tipo de habitação e área de residência. Os autores concluíram que o índice de status socioeconômico que criaram era uma medida conveniente, mas imperfeita, dos atributos que acreditavam estar associados com status, insistindo que não levava em consideração "muito da significante vida das pessoas" (Warner et al., 1960, p. 252). O desenvolvimento de medidas de status por atributo, baseadas nas ocupações 
e não nas famílias, tornou-se foco central da pesquisa em estratificação nos anos seguintes. ${ }^{3}$

Nesse processo, no entanto, a interpretação do status sofreu uma mudança gradual, partindo das avaliações normativas de valor para medidas posicionais de vantagens socioeconômicas.

\section{Gradações posicionais}

Na década de 1960, a insatisfação com o funcionalismo normativo estimulou o desenvolvimento de teorias alternativas de estratificação. Na tentativa de transcender o que percebia como o foco unilateral do marxismo na classe econômica e no conflito e a ênfase igualmente unilateral do funcionalismo nos valores compartilhados e no consenso, Gerhard Lenski (1984) propôs uma teoria de estratificação "sintética", baseada em uma leitura peculiar da obra de Max Weber sobre classe, status e partido. Lenski defende que Weber desenvolveu uma visão sintética da estratificação na qual classe, como recursos econômicos, status, como avaliações de superioridade e inferioridade, e partido, definido como poder político, eram três dimensões da desigualdade social. Essas dimensões, segundo Lenski, forneciam ferramentas conceituais mais completas para se entender a desigualdade do que aquelas oferecidas pelas teorias marxista e durkheimiana.

No entanto, Weber não concebia a estratificação em termos de dimensões separadas, nem conceitualizou classes

\footnotetext{
${ }^{3} \mathrm{O}$ termo status por atributo [attributional status] foi desenvolvido por Marriott (1959). Diz respeito a hierarquias de status baseadas em alguns poucos indicadores externos e facilmente reconhecíveis. Marriott sustenta que o status por atributo é encontrado em grandes comunidades onde a maioria das relações é anônima, onde as sanções pela violação das expectativas relacionadas a um status não podem ser impostas e onde ter a garantia da colaboração dos outros não é essencial para o acesso a recursos e oportunidades valorizados. O status por atributo tem como contraponto o status interacional, que surge da troca de mercadorias e serviços no contexto de relações diretas e pessoais. Essa distinção é interessante, mas o significado do status por atributo em sua relação com as teorias da desigualdade nunca foi esclarecido por Marriott ou por outros que o utilizaram (ver Lockwood, 1992; Plowman et al., 1962).
} 
e grupos de status como gradações. Para Weber, status, classe e partido eram modos de formação de grupos relacionados com os conflitos sobre a distribuição do poder social. A origem mais plausível para os conceitos de status como gradação e para a metáfora espacial frequentemente associada a eles é a ideia de espaço social de Pitirim Sorokin. Em Social and cultural mobility, Sorokin sustenta que a sociedade pode ser representada como um espaço bidimensional, no qual a dimensão vertical é uma ordenação de status baseada na "distribuição desigual de direitos e privilégios" e a horizontal se refere a diferenças nos grupos sem conotação hierárquica inerente (Sorokin, 1964, p. 11). A metáfora de Sorokin deu inspiração para conceitos posicionais de status e para grande parte da pesquisa contemporânea em mobilidade e realização de status [status attainment], cujo interesse principal era analisar o movimento dos indivíduos ao longo de um espaço social contínuo. A influência conjunta de 52 Weber e Sorokin é explícita em Lenski:
"Outro pioneiro do movimento sintetizante foi Pitirim Sorokin. Seu trabalho mais antigo, Social mobility, é provavelmente o primeiro tratamento extensivo e sistemático sobre estratificação social onde a perspectiva sintética é dominante. [...] Isso se manifesta principalmente na utilização da visão multidimensional da estratificação, uma tendência comum no trabalho de síntese, evidente também na obra de Weber" (Lenski, 1984, p. 18).

Essa interpretação multidimensional de Weber, combinada com a metáfora espacial de Sorokin, proporcionou a estrutura teórica necessária para a conceitualização do status como uma hierarquia de posições baseada em recursos socioeconômicos. Embora os termos status e prestígio continuassem a ser utilizados por Lenski como sinônimos para se referir às avaliações de superioridade e inferioridade, a dimensão 
do status foi, na verdade, completamente eliminada como um fator explicativo independente em seu modelo de estratificação social. Ao explicar a razão da pouca atenção despendida ao status em sua obra sobre poder e privilégio, Lenski argumentou que "o prestígio pode ser entendido mais facilmente como uma função do poder e do privilégio do que o contrário" (Lenski, 1984, p. 430). Longe de chegar a uma abordagem mais equilibrada sobre a estratificação social, a síntese de Lenski entre Marx e Durkheim resultou na eliminação do status como avaliações sociais de superioridade e inferioridade. Essa eliminação teve consequências, já que a sua leitura de Weber se tornou uma referência bastante citada como justificativa teórica para o emergente conceito de status como posição socioeconômica (ver Blishen e Carroll, 1982; Haug, 1977; Nam e Terrie, 1982; Powers, 1982).

Quase ao mesmo tempo, várias medidas de status ocupacional foram sendo desenvolvidas como forma de localizar posições na estrutura social. As três principais abordagens foram as escalas de prestígio ocupacional de North-Hatt baseadas em avaliações coletivas (Reiss, 1961), as medidas de status socioeconômico baseadas nas de prestígio (Duncan, 1961) e as medidas de status socioeconômico que combinavam vários atributos das ocupações (Blishen, 1958; Nam e Terrie, 1982). Embora essas escalas sejam altamente correlacionadas, as diferenças no modo pelo qual são construídas levam a interpretações muito diferentes. A interpretação das medidas puras de status socioeconômico é geralmente bem direta: não é baseada em avaliações sociais e simplesmente mede a posição na estrutura social de acordo com vários atributos das ocupações. Já o significado das escalas de prestígio ocupacional é mais ambíguo: como se baseiam em avaliações feitas pelas pessoas em geral, as ordenações de prestígio ocupacional necessariamente refletem representações coletivas sobre algumas características das ocupações. Qual dessas características está por trás das hie- 
rarquias, no entanto, nunca ficou muito claro, muito embora as principais posições sejam bastante conhecidas.

De um lado, os sociólogos que enfatizavam a natureza consensual dos rankings ocupacionais afirmavam que eles refletiam a existência de um quadro normativo compartilhado, no sentido durkheimiano, que estrutura as relações sociais e garante a integração social. Essa posição é bem articulada por Shils (1975), que defende que as posições de ranking mais elevado nas escalas de prestígio são aquelas que estão mais próximas do centro da sociedade. As ocupações de ranking mais baixo são aquelas cujos membros contribuem pouco para a criação e a expressão da ordem social moral, cognitiva e material. A alta correlação entre rankings ocupacionais e características socioeconômicas das ocupações é interpretada como evidência de que é a proximidade com os princípios mais elevados de uma ordem transcendente que dá a algumas ocupações alto prestígio, e não as 54 recompensas materiais ou o poder terreno ligado a elas.

Como o funcionalismo normativo, com seu pressuposto de que são as normas e os valores os elementos que comandam as relações sociais, perdia rapidamente sua força no pensamento sociológico, essa explicação nunca foi muito popular entre os sociólogos que projetavam, analisavam e utilizavam escalas de prestígio. Seguindo Lenski, outros autores afirmaram que as escalas de prestígio ocupacional de fato constituem um quadro normativo que reflete o valor das ocupações, mas acrescentaram que esse quadro é inteiramente determinado pelo poder e por vantagens materiais. Em Occupational prestige in comparative perspective (1977), por exemplo, Donald Treiman argumenta que as escalas de prestígio ocupacional refletem uma percepção normativa da estratificação social válida universalmente e avaliada consensualmente:

"O ponto importante é que a avaliação é moral, o que traz uma conotação acerca do valor relativo do atributo que 
está sendo julgado, e não é simplesmente uma questão de gosto ou preferência. Nesse sentido, comparações invejosas ${ }^{4}$ refletem normas e valores compartilhados a respeito da posição relativa de atributos nas hierarquias de valor. A moeda do valor moral é o prestígio, conhecido também como honra, consideração, respeito, posição e estima" (Treiman, 1977, p. 20).

Seguindo de perto o argumento padrão funcionalista, Treiman sustenta que todas as sociedades, do passado e do presente, possuem os mesmos imperativos funcionais, que resultam em padrões similares de diferenciação social e, por isso, em distribuições similares de poder e privilégio. No entanto, em oposição ao funcionalismo normativo, para o qual o sistema de valor determina tanto a ordenação das posições quanto as recompensas materiais relacionadas a cada uma, Treiman defende que é o poder e as recompensas que determinam seu prestígio (Treiman, 1977, p. 17). Ao fazer isso, o autor coloca o argumento funcionalista normativo de ponta-cabeça, parafraseando a inversão da dialética hegeliana feita por Marx: ao invés de poder e classe estarem subsumidos pelo status, status se torna uma categoria residual quase inteiramente determinada pela posição socioeconômica.

A terceira posição, que hoje ganha amplo consenso, é que as escalas de prestígio ocupacional, como as medidas puras de status socioeconômico, medem a posição na estrutura social e não status no sentido de avaliações de superioridade e inferioridade (DiMaggio e Mohr, 1985; Featherman et al., 1975; Featherman e Hauser, 1976; Goldthorpe e Hope, 1974; Hope, 1982; Sørensen, 1994; Wegener, 1992).

\footnotetext{
${ }^{4}$ N.T.: Essa expressão, no original "invidious comparisons", remete diretamente à obra de Thorstein Veblen e a seu livro The theory of the leisure class, de 1899. Nas edições dessa obra em português, com a tradução realizada por Olivia Krähenbühl, o mesmo termo "invidious" nessa expressão foi traduzido como "invejosa", "odiosa", "ofensiva e injusta", "injusta”, "impiedosa”, "injuriosa”, "desagradável” e "de competição".
} 
Essa posição admite que status nesse último sentido tem importância para as relações sociais, mas defende que não é isso que as escalas de prestígio ocupacional medem.

A variação comumente mais aceita desta posição, proposta por Goldthorpe e Hope (1974), é que as escalas de prestígio ocupacional mensuram a "desejabilidade" ou o "encanto" [goodness] das ocupações pelas suas recompensas socioeconômicas. Para os autores, as pessoas geralmente têm consciência das vantagens culturais e materiais relacionadas às ocupações. ${ }^{5}$ Nas mesmas condições, concordam que as posições que oferecem maiores recompensas são mais desejáveis do que aquelas que oferecem menores. Desse modo, as escalas de prestígio ocupacional são interpretadas como avaliações factuais das várias vantagens da posição social e não mais como avaliações normativas baseadas em valor.

Mais recentemente, tem sido argumentado que as escalas de prestígio ocupacional deveriam ser interpretadas como 56 uma representação unidimensional de uma estrutura bidimensional, cujas dimensões centrais são os recursos econômicos e culturais (Grusky e Van Rompaey, 1992). Evidências que sustentam esse argumento são encontradas na obra de Bourdieu (1979), que conceitua a estrutura ocupacional como um espaço bidimensional com o capital cultural e o econômico como suas duas principais dimensões, no trabalho empírico de DeGraff, Ganzeboom e Kalmijn (1989), que operacionaliza e testa a utilidade dessas duas dimensões para analisar estilos de vida, e mesmo no resultado encontrado por Duncan (1961) de que o prestígio ocupacional representa uma combinação entre renda e educação.

Uma interpretação ligeiramente diferente é proposta por Hope (1982), que defende que as escalas de prestígio

\footnotetext{
${ }^{5}$ De fato, os valores na escala ocupacional apresentam maior associação com a renda e a educação tal como percebidas pelas pessoas do que com seus valores reais. Renda e educação percebidas explicam juntas "mais de $93 \%$ da variação do prestígio geral' (Powell e Jacobs, 1984, p. 180).
} 
ocupacional refletem tanto as recompensas materiais relacionadas às ocupações quanto a utilidade percebida de cada uma para a sociedade. Usando escalonamento multidimensional, Grasmick (1976) mostra, nessa mesma direção, que os vetores, medindo a posição social das ocupações, refletem uma combinação entre recompensas materiais e valor para a sociedade, embora essa última dimensão opere apenas na faixa intermediária da escala da primeira dimensão. É interessante como ocupações que estão em polos opostos na dimensão de valor social de Grasmick são bastante parecidas com aquelas que Bourdieu posicionou em polos opostos em sua dimensão de capital cultural-econômico (Bourdieu, 1979, pp. 140-141; Grasmick, 1976, p. 101). Em ambos os casos, a faixa intermediária do espaço ocupacional é caracterizada por uma oposição entre ocupações como professores e assistentes sociais, de um lado, e ocupações mais comerciais, tais como pequenos comerciantes e gerentes, de outro. Desse modo, valor para a sociedade e capital cultural parecem ser interpretações um pouco diferentes para a mesma dimensão do espaço ocupacional. Tanto Goldthorpe e Hope (1974) quanto Hope (1982) reconhecem que o conceito que utilizam conserva um componente avaliativo, mas sustentam que esse componente não tem conotação normativa nem significância na legitimação da desigualdade social.

Independente de como as duas dimensões são interpretadas, as escalas de prestígio ocupacional são conceitualizadas como gradações sintéticas, combinando atributos econômicos e socioculturais - com definições as mais variadas - das ocupações. São interpretadas como medidas posicionais das vantagens materiais e simbólicas que acompanham as ocupações, e não como avaliações normativas de valor. Nesse sentido, estão muito mais próximas do conceito weberiano de classe, como possibilidades no mercado, do que de status, como reivindicações de estima social 
(ver DiMaggio e Mohr, 1985; Featherman et al., 1975; Featherman e Hauser, 1976; Goldthorpe e Hope, 1974; Hope, 1982; Nam e Terrie, 1982; Ollivier, 2000; Sørensen, 1994; Wegener, 1992).

A rejeição às interpretações normativas para o prestígio ocupacional geralmente reside em dois tipos diferentes de argumentos. O primeiro se baseia no resultado empírico que indicou que, quando as pessoas são perguntadas diretamente sobre o que têm em mente quando avaliam as ocupações, poucas mencionam critérios normativos que dizem respeito ao valor dessas ocupações (Coleman e Rainwater, 1978; Goldthorpe e Hope, 1972 e 1974; Reiss, 1961). A grande maioria menciona fatores socioeconômicos, como educação, renda e outros benefícios (Reiss, 1961, p. 34). O outro argumento, que tem implicações teóricas mais profundas, envolve a rejeição de um dos pressupostos centrais para o funcionalismo normativo, qual seja, o de que um 58 quadro normativo que abarque toda a sociedade é essencial para a integração social.

Ao longo dos anos, os sociólogos questionaram progressivamente se, nas sociedades de mercado avançadas, é possível que uma única e vasta ordem de status pudesse permear a totalidade da vida social e exercer uma influência sistemática nas relações sociais. Essa posição se fundamenta em dois argumentos inter-relacionados. O primeiro é que as sociedades contemporâneas são altamente complexas e segmentadas, de tal modo que as pessoas possuem filiações sociais múltiplas que não necessariamente coincidem (Blau e Schwartz, 1984; DiMaggio e Mohr, 1985; Faunce, 1990). Complexidade e segmentação contribuem para a emergência de culturas de status múltiplas e locais as quais as pessoas aprendem seletivamente a mostrar nos contextos sociais apropriados (DiMaggio, 1987; Erickson, 1996). Esses sistemas locais de status podem ser mais relevantes para aspectos específicos da vida cotidia- 
na das pessoas do que uma única ordenação de status das ocupações. $^{6}$

O segundo argumento é que as sociedades contemporâneas são caracterizadas pela proliferação de relações indiretas e anônimas (Calhoun, 1991; Lockwood, 1992; Marriott, 1959; Parkin, 1979; Scott, 1996). Relações indiretas são mediadas por estruturas de larga escala, como organizações burocráticas, o mercado e a mídia de massa (Calhoun, 1991). E se referem a um domínio da experiência que é distinto do mundo experienciado através de relações diretas, estabelecidas face a face. Meu argumento é que as escalas de prestígio ocupacional devem ser consideradas como uma manifestação da distinção entre esses dois aspectos da experiência social. Elas se formam parcialmente baseadas em relações diretas, mas também baseadas, em grande medida, pela experiência indireta fornecida pela mídia de massa (Ollivier, 2000). A extensão da ruptura entre relações diretas e indiretas está aberta a debates (Herness, 1991), mas é razoável supor que, se as escalas de prestígio ocupacional são formadas com base em relações indiretas, possuem também uma capacidade real, mesmo que limitada, de organizar as relações face a face das pessoas em suas vizinhanças, nos seus ambientes de trabalho e dentro de suas redes de amigos e conhecidos. ${ }^{7}$

Juntos, esses argumentos sugerem que as sociedades contemporâneas avançadas compreendem ordens normativas múltiplas, sobrepostas e possivelmente em conflito, nas quais o status, como avaliações de superioridade e inferioridade,

\footnotetext{
${ }^{6}$ Ver, por exemplo, Matsueda et al. (1992) sobre subculturas criminais, Milner (1996) sobre status entre estudantes secundários e Ollivier (2006) sobre prestígio na música popular.

${ }^{7}$ Este argumento é uma resposta à tão discutida, porém subteorizada, distinção feita por Marriott (1959) entre status por atributo e interacional. Marriott desenvolveu esses conceitos para dar conta da discrepância entre as hierarquias oficiais das castas na Índia e as hierarquias de castas que ele encontrou em nível local. Para Marriott, essas hierarquias locais estavam fundamentadas na troca direta de bens e serviços.
} 
opera predominantemente - embora não exclusivamente - nos níveis micro e local: em pequenas comunidades (Featherman, Jones e Hauser, 1975; Parkin, 1979), entre diferentes frações de classes e grupos (Coleman e Rainwater, 1978; Hope, 1982; Milner, 1996; Ostrower, 1995), entre grupos ocupacionais adjacentes ou competidores (Blau e Duncan, 1967, pp. 63-70), ou dentro de várias esferas de status e estilo de vida (Hall, 1992). Ao invés de ser concebido como um quadro normativo global integrando a sociedade como um todo, status como avaliação coletiva seria melhor conceitualizado operando principalmente em nível local, dentro de comunidades relativamente pequenas e em contextos sociais específicos.

\section{0 conceito de status categórico de Weber}

Como alternativa ao funcionalismo normativo, o conceito de grupos de status de Weber tem sido extremamente influente na sociologia. Diferentemente de Marx e Engels

60 (1967), que pensavam que ideologias e privilégios baseados em status estavam sendo dissolvidos pelas relações de mercado, e de Durkheim (1960a), que estava mais interessado nas rupturas de legitimidade do que nos mecanismos que geram a desigualdade, Weber considerava que as avaliações de superioridade e inferioridade têm consequências fundamentais para o entendimento da desigualdade social. Defendendo que o status poderia se basear em qualquer critério que separasse convenientemente grupos uns dos outros, Weber enfatizou a natureza arbitrária das reivindicações de status e seu papel nos processos de dominação. E por meio do conceito de fechamento social, Weber também forneceu um mecanismo pelo qual as reivindicações de status resultam em dominação e desigualdade social.

O conceito de Weber combina os aspectos avaliativos e posicionais do status. Refere-se tanto às "reivindicações bem-sucedidas de estima social" expressas em estilos de vida distintivos quanto às posições sociais obtidas com base 
nessas reivindicações. Assim, status diz respeito tanto aos privilégios costumeiros e jurídicos, que criam e mantêm as desigualdades, quanto às avaliações de superioridade e inferioridade, que os legitima. É uma modalidade de formação de grupos que representa um dos três tipos ideais de agrupamentos sociais: grupos de status são grupos reais formados com base em estimativas coletivas de honra; classes econômicas são grupos indefinidos de pessoas que compartilham recursos econômicos similares; partidos são associações instrumentais interessadas em poder político. Status é também um modo de distribuição do poder social. Em oposição às classes, cujos membros usam seus recursos para ganhar vantagens nos mercados abertos, os grupos de status tipicamente buscam o poder através do fechamento costumeiro ou jurídico, que lhes permite restringir a entrada de membros no grupo e ganhar acesso exclusivo a oportunidades e recompensas. O exemplo supremo da estratificação por status, sobre o qual Weber construiu seu conceito, é o dos estados ou estamentos [estates] nobres do início do período moderno, cujas reivindicações de status eram expressas em estilos de vida distintivos, cujo poder estava legitimado por uma ideologia de superioridade inata e cujos privilégios eram garantidos pela autoridade do Estado. O conflito entre os estamentos nobres e a burguesia emergente foi visto por Weber como um conflito entre status e classe, entre formas alternativas de distribuição do poder social. Os estamentos utilizavam de sua autoavaliada superioridade para legitimar restrições sobre o livre mercado, enquanto a classe burguesa exercia seu poder por meio do mercado, em virtude do controle que possuía sobre recursos limitados (Weber, 1978, p. 937).

No entanto, devido a sua natureza fragmentada e ao fato de que foi elaborado no contexto do declínio do poder dos estamentos nobres, o conceito weberiano tem várias deficiências para o estudo do status nas sociedades contem- 
porâneas. Em primeiro lugar, Weber conceitua status principalmente como uma modalidade de formação de grupo e não discute as situações em que o status é um atributo de indivíduos formalmente iguais dentro de uma comunidade. Por exemplo, a competição por status entre os homens era um traço central da vida social nas sociedades tradicionais da Nova Guiné. Como essas sociedades não tinham transmissão hereditária do status, apenas pouca acumulação privada dos recursos entre gerações, e não possuíam uma autoridade firmada, o status permanecia em linhas gerais como uma questão para a competição entre os indivíduos e não resultava na formação de grupos de status estáveis (Forge, 1970). Da mesma forma, nas sociedades democráticas liberais contemporâneas, o ideal meritocrático de que as recompensas devem ser concedidas principalmente com base no talento e no esforço individuais dificulta que privilégios e exclusões se legitimem a partir de critérios coletivos ou inscritos como 62 linhagem, casta, etnia ou gênero (Murphy, 1988; Parkin, 1979). Uma segunda dificuldade é que o conceito de Weber se baseia no pressuposto de que o status, como avaliações de superioridade e inferioridade, tipicamente resulta em exclusão costumeira ou jurídica, restringindo a operação do mercado. Embora esse pressuposto seja apropriado para grupos, como estamentos e castas, ele não abre espaço para a possibilidade de que as avaliações de superioridade e inferioridade se tornem recursos utilizados pelos indivíduos para ganhar vantagens através do mercado, e não contra.

Nas sociedades contemporâneas, onde indivíduos formalmente iguais competem entre si em várias arenas, a associação de status com grupos corporativos e com fechamento formal é especialmente problemática. As avaliações de superioridade e inferioridade são mais frequentemente usadas como recursos em disputas formalmente abertas do que como meios de legitimar monopólios legais de grupos corporativos (DiMaggio, 1982, p. 190; Douglas, 1979, p. 193; 
Murphy, 1988, p. 222; Parkin, 1979, p. 65). Nesse sentido, status se refere a hierarquias socialmente construídas de qualidades, atributos e realizações ao invés de a uma única hierarquia de subgrupos em uma comunidade (DiMaggio e Mohr, 1985; Murphy, 1988). Os indivíduos ganham status ao mostrar a posse de, ou a familiaridade com, qualidades e atributos que são altamente valorizados em determinados períodos e locais. Exibir sinais apropriados de alto status tem sido mostrado como capaz de abrir oportunidades em todos os tipos de situações, incluindo em escolas (Bourdieu e Passeron, 1977; Collins, 1979; DiMaggio e Mohr, 1985), no mercado de trabalho (Collins, 1979), na competição por publicação acadêmica (Merton, 1968; Powell, 1985), na seleção conjugal (DiMaggio e Mohr, 1985), em pequenos grupos orientados para a execução de tarefas (Webster e Driskell, 1978) e em mercados de bens e serviços (Podolny, 1993). As vantagens associadas incluem maior reconhecimento em níveis de conquista semelhantes (Merton, 1968; Webster e Driskell, 1978), menores custos e maiores rendimentos em transações comerciais (Podolny, 1993), acesso a círculos sociais restritos (Ostrower, 1996) e maior oportunidade de participar de interações sociais (Webster e Driskell, 1978).

No entanto, essa transição do fechamento coletivo para a competição individual não significa que o status seja simplesmente o reflexo do talento individual e dos esforços nas atividades que beneficiam uma coletividade (Homans, 1950). As hierarquias de atributos, qualidades e realizações em que o status se baseia não são dadas naturalmente. São construídas socialmente e, como tais, são susceptíveis à manipulação por grupos sociais poderosos. Nas sociedades contemporâneas, indivíduos formalmente iguais permanecem diferenciados e estratificados de acordo com diversos critérios, incluindo classe econômica, origem étnica e gênero. Classes e grupos cujos membros ocupam postos-chave nas instituições sociais que validam as hierarquias 
de status, como escolas, governos e mídia, estão em posição de fazer com que as qualidades e os atributos de seus próprios grupos sejam percebidos como medidas universais de superioridade (Bourdieu, 1979; Parkin, 1971). ${ }^{8}$ A manipulação das hierarquias de status é um meio pelo qual os grupos preservam as vantagens coletivas ao longo do tempo, na ausência da exclusão coletiva sancionada legalmente. A monopolização das oportunidades no mercado, baseada em atributos individuais, e a monopolização coletiva por meio de regras formais de exclusão muitas vezes produzem resultados parecidos, mas é mais fácil legitimar a primeira do que a segunda nas sociedades meritocráticas contemporâneas (Parkin, 1979, p. 65).

Apesar da ênfase colocada por Weber na estrita associação entre os aspectos avaliativo e jurídico do status, hoje os sociólogos tendem a dissociá-los e a ver o status como um conceito que varia ao longo de algumas dimensões (Brint, 64 1996; Clark, 1995; Milner, 1994 e 1996; Turner, 1988). Em ambientes individualistas e competitivos, em particular, o status jurídico pode ser legitimado em bases distintas das avaliações coletivas de superioridade e inferioridade. Inversamente, status neste último sentido não resulta necessariamente em fechamento coletivo sancionado legalmente e na criação de grupos de status estáveis.

Monopólios legais, como aqueles desfrutados pelos estamentos nobres e pelas castas, não foram eliminados, mas as estratégias de fechamento legal estão limitadas a situações específicas. Quando esses monopólios são legitimados pela referência a reivindicações de superioridade, é geralmente com base em contribuições realizadas aos objetivos sociais

\footnotetext{
${ }^{8}$ Mesmo que Bourdieu tenha exagerado na habilidade das classes dominantes para impor seus próprios atributos como medidas universais de distinção (Hall, 1992), a vantagem advinda do controle sobre as posições institucionais não deve ser subestimada da mesma forma.
} 
valorizados, e não com base em características coletivas inscritas (Murphy, 1988). Por exemplo, as associações profissionais procuram garantir direitos exclusivos sobre a prática em suas atividades baseando-se nas habilidades e conhecimentos especiais, assim como artistas em mercados restritos almejam a imposição pelo Estado de quotas sobre produtos culturais com base na contribuição especial que fazem à manutenção da identidade nacional (Ollivier, 2006). Cidadania é outro exemplo de status jurídico. Por meio dela são definidos os direitos e deveres dos cidadãos dentro de um Estado-nação, sem recorrer a ideias de superioridade e inferioridade para sua legitimidade (Marshall, 1964). Conforme observou Marshall, a cidadania baseada no princípio da igualdade formal arruinou os privilégios dos estamentos nobres, mas se tornou também uma plataforma para grupos desprivilegiados que buscavam legitimar suas reivindicações contra o Estado-nação e equilibrar suas desvantagens no mercado. Seguindo o argumento de Marshall, Halsey (1986) percebe as sociedades contemporâneas divididas entre dois princípios distributivos conflitantes: os de "classe operando através dos mercados de trabalho e de capital, e status operando através dos sistemas político e jurídico na definição dos direitos dos cidadãos" (Halsey, 1986, p. 61). Desse modo, status, nesse sentido legal, permanece importante para a compreensão de aspectos da desigualdade, mas muita confusão seria evitada se o termo status jurídico [ou legal] fosse utilizado referindo-se a esse sentido específico.

\section{Conclusão}

Em que medida os clássicos conceitos de status contribuem para nosso entendimento dos aspectos simbólicos da desigualdade nas sociedades contemporâneas? Não muito, na minha opinião. Os conceitos funcionalistas normativos fazem fortes asserções sobre o papel das avaliações coletivas para a estabilidade e a integração sociais. No entanto, 
o pressuposto por trás dessas asserções é insustentável à luz da complexidade, da segmentação e do anonimato das relações sociais nas sociedades contemporâneas. As gradações posicionais, originalmente desenvolvidas como uma extensão do funcionalismo normativo, são úteis na pesquisa empírica porque sintetizam a posição social ao longo de várias dimensões da desigualdade. No entanto, não dizem nada a respeito das avaliações de superioridade e inferioridade assim como sobre qualquer mecanismo gerador das desigualdades. As gradações posicionais são medidas nominais de posição social e são compatíveis com uma ampla variedade de perspectivas teóricas. O conceito categórico de Weber continua útil para a compreensão de como os grupos se utilizam de reivindicações de superioridade para legitimar a exclusão costumeira ou legal. No entanto, o conceito weberiano não é muito útil para compreendermos como o status leva à exclusão informal em ambientes pouco diferencia66 dos, em que os indivíduos são formalmente iguais, as classificações apresentam frágil institucionalização e existem poucas restrições aos relacionamentos sociais. Neste ponto, os conceitos que apresentam o status como um atributo dos indivíduos poderiam se mostrar mais úteis, mas um exame pormenorizado de seus pressupostos e limitações está além do escopo deste artigo.

Assim, a sociologia precisa de conceitos de status que sejam, ao mesmo tempo, menos e mais ambiciosos que os conceitos clássicos desenvolvidos por Durkheim e Weber. Menos ambiciosos porque o status precisa ser conceitualizado não como um quadro integrativo de abrangência total, mas como um mosaico de hierarquias e estratégias múltiplas. E mais ambiciosos porque é mais difícil entender como o status opera abaixo da superfície das relações sociais entre indivíduos formalmente iguais do que como um meio de impor fronteiras bem visíveis e reconhecidas publicamente entre grupos. 


\section{Michèle Ollivier}

é professor da University of Ottawa

\section{Referências bibliográficas}

BARBER, B. 1957. Social stratification: a comparative analysis of structure and process. New York: Harcourt, Brace, and World.

BLAU, P. 1967. Exchange and power in social life. New York: John Wiley \& Sons.

BLAU, P. 1971. Bureaucracy in modern society. New York: Random House.

; DUNCAN, O. D. 1967. The American occupational structure. New

York: Wiley.

; SCHWARTZ, J. E. 1984. Crosscutting social circles. Orlando: Academic Press.

BLISHEN, B. 1958. "The construction and use of an occupational class scale”. Canadian Journal of Political Science, vol. 24, pp. 519-531.

BLISHEN, B.; CARROLL, W. K. 1982. "Socioeconomic measures from Canadian Census data”. In: POWERS, M. G. (ed.). Measures of socioeconomic status. Boulder, Colorado: Westview Press.

BOURDIEU, P. 1977. Outline of a theory of practice. Cambridge: Cambridge University Press.

. 1979. La distinction: critique sociale du jugement. Paris: Les Éditions de Minuit.

; COLEMAN, J. S. (eds.). 1991. Social theory for a changing society. Boulder: Westview Press.

; PASSERON, J. C. 1977. Reproduction in education, society, culture. Beverly Hills: Sage Publications.

BRINT, S. 1996. The status of the status group concept. Trabalho apresentado no Encontro Anual da American Sociological Association, New York.

CALHOUN, C. 1991. "Indirect relationships and imagined communities: large-scale social integration and the transformation of everyday life". In: BOURDIEU, P.; COLEMAN, J. S. (eds.). Social theory for a changing society. Boulder: Westview Press.

CLARK, S. 1995. State and status: the rise of the state and aristocratic power in Western Europe. Montreal and Kingston: McGill-Queen's University Press.

COLEMAN, R. P.; RAINWATER, L. 1978. Social standing in America. New York: Basic Books.

COLLINS, R. 1979. The credential society. New York: Academic Press.

DAVIS, K.; MOORE, W. E. 1945. "Some principles of stratification”. American Sociological Review, vol. 10, pp. 15-21. 
DEGRAFF, P. M.; GANZEBOOM, H. B. G.; KALMIJN, M. 1989. "Cultural and economic dimensions of occupational status". In: JANSEN, W.; DRONKERS, J.; VERRIPS, K. (eds.). Similar or different? Amsterdam: Siswo.

DiMAGGIO, P. 1982. "Cultural capital and school success: the impact of status culture participation in the grades of US high school students". American Sociological Review, vol. 47, pp. 189-201.

1987. "Classification in art". American Sociological Review, vol. 52, pp. $440-455$.

; MOHR, J. 1985. "Cultural capital, educational attainment, and marital selection". American Journal of Sociology, vol. 90, $\mathrm{n}^{\circ}$ 6, pp. 1.231-1.261.

DOUGLAS, M. 1979. In the active voice. London: Routledge \& Kegan Paul.

DUNCAN, O. D. 1961. "A socio-economic index for all occupations”. In: REISS, A. J. (ed.). Occupations and social status. New York: Free Press, pp. 109-138.

DURKHEIM, E. 1960a. Le suicide. Paris: Presses Universitaires de France. 1960b. De la division du travail social. Paris: Presses Universitaires de France.

ERICKSON, B. 1996. "Culture, class, and connections". American Journal of Sociology, vol. 102, $\mathrm{n}^{\circ} 1$, pp. 217-235.

FAUNCE, W. F. 1990. "On the meaning of occupational status: implications for stratification theory and research". Sociological Focus, vol. 23, $\mathrm{n}^{\circ} 4$, pp. 267-285.

FEATHERMAN, D. L.; HAUSER, R. M. 1976. "Prestige or socioeconomic scales in the study of occupational achievement?". Sociological Methods and Research, vol. 4, $\mathrm{n}^{\circ}$ 4, pp. 403-422.

; JONES, F. L.; HAUSER, R. M. 1975. "Assumptions of social mobility research in the U.S.: the case of occupational status". Social Science Research, vol. 4, pp. 329-360.

FORGE, A. 1970. "Prestige, influence, and sorcery: a New Guinea example”. In: DOUGLAS, M. (ed.). Witchcraft accusations and confessions. London: Tarrstock.

GOLDTHORPE, J. H.; HOPE, K. 1972. "Occupational grading and occupational prestige”. Social Science Information, vol. 11, nº 5, pp. 17-73.

1974. The social grading of occupations: a new approach and scale. Oxford: Clarendon Press.

GRASMICK, H. G. 1976. "The occupational prestige structure: a multidimensional scaling approach”. The Sociological Quarterly, vol. 17, pp. 98-108. 
GRUSKY, D. B. (ed.). 1994. Social stratification: class, gender and race in sociological perspective. Boulder: Westview Press.

; VAN ROMPAEY, S. E. 1992. "The vertical scaling of occupations: some cautionary comments and reflections". American Journal of Sociology, vol. 97, pp. 1.712-1.728.

HALL, J. R. 1992. "The capital(s) of culture: a nonholistic approach to status situations, class, gender, and ethnicity". In: LAMONT, M.; FOURNIER, M. (eds.). Cultivating differences: symbolic boundaries and the making of inequality. Chicago and London: The University of Chicago Press.

HALSEY, A. H. 1986. Change in British society. Oxford: Oxford University Press.

HAUG, M. R. 1977. "Measurement in social stratification". Annual Review of Sociology, vol. 3, pp. 51-77.

HERNESS, G. 1991. “Comments”. In: BOURDIEU, P.; COLEMAN, J. S. (eds.). Social theory for a changing society. Boulder: Westview Press.

HOMANS, G. 1950. The human group. New York: Harcourt, Brace and Company.

HOPE, K. 1982. "A liberal theory of prestige". American Journal of Sociology, vol. 87, $\mathrm{n}^{\circ}$ 5, pp. 1.011-1.031.

LAMONT, M.; FOURNIER, M. (eds.). 1992. Cultivating differences: symbolic boundaries and the making of inequality. Chicago and London: The University of Chicago Press.

LENSKI, G. E. 1984. Power and privilege: a theory of social stratification. Chapel Hill: The University of North Carolina Press.

LOCKWOOD, D. 1992. Solidarity and schism. Oxford: Clarendon Press.

MARRIOTT, M. 1959. "Interactional and attributional theories of caste ranking”. Man in India, vol. 39, nº 2, pp. 92-107.

MARSHALL, T. H. 1964. Class, citizenship, and social development. Chicago: The University of Chicago Press.

MARX, K.; ENGELS, F. 1967. The communist Manifesto. Clinton: Random House.

MATSUEDA, R. L.; GARTNER, R.; PILIAVIN, I.; POLAKOWSKI, M. 1992. "The prestige of criminal and conventional occupations: a subcultural model of criminal activity". American Sociological Review, vol. 57, $\mathrm{n}^{\circ} 6$, pp. 752-770.

MERTON, R. K. 1968. "The Matthew effect in science”. Science, vol. 159, pp. 56-63.

MILNER, M. 1994. A general theory of status relations and an analysis of Indian culture. New York, Oxford: Oxford University Press. 
1996. Geeks, weirdos, and cool kids: status among American adolescents. Trabalho apresentado no Encontro Anual da American Sociological Association, New York.

MURPHY, R. 1988. Social closure: the theory of monopolization and exclusion. Oxford: Clarendon Press.

NAM, C. B.; TERRIE, E. W. 1982. "Measurement of socioeconomic status from United States census data”. In: POWERS, M. G. (ed.). Measures of socioeconomic status. Boulder, Colorado: Westview Press.

OLLIVIER, M. 2000. "Too much money off other people's backs: status and boundaries in postindustrial society". Canadian Journal of Sociology, vol. $25, \mathrm{n}^{\circ} 4,441-470$.

. 2006. "Snobs and quétaines: prestige and boundaries in popular music in Quebec". Popular Music, vol. 25, n 1, pp. 97-116.

OSSOWSKI, S. 1963. Class structure in the social consciousness. New York: Free Press.

OSTROWER, F. 1995. Why the wealthy give: the culture of elite philanthropy. Princeton: Princeton University Press. 1996. Status and elite philanthropy: hierarchies and processes. Trabalho apresentado no Encontro Anual da American Sociological Association, New York.

70 PARKIN, F. 1971. Class inequality and political order. London: MacGibbon and Kee.

1979. Marxism and class theory: a bourgeois critique. London: Tavistock.

PARSONS, T. 1954. "An revised analytical approach to the theory of social stratification”. In: PARSONS, T. Essays in sociological theory. New York: Free Press.

PLOWMAN, D. E. G.; MINCHINTON, W. E.; STACEY, M. 1962. "Local social status in England and Wales". The Sociological Review, vol. 10, $\mathrm{n}^{\circ}$ 2, pp. 161-195.

PODOLNY, J. 1993. "A status-based model of status competition". American Journal of Sociology, vol. $98, \mathrm{n}^{\circ}$ 4, pp. 829-872.

POWELL, W. W. 1985. Getting into print: the decision-making process in scholarly publishing. Chicago: Chicago University Press.

POWELL, B.; JACOBS, J. 1984. "Gender differences in the evaluation of prestige”. The Sociological Quarterly, vol. 25, n 2, pp. 173-190.

POWERS, M. G. (ed.). 1982. Measures of socioeconomic status. Boulder, Colorado: Westview Press.

REISS, A. J. (ed.). 1961. Occupations and social status. New York: Free Press. 
SCOTT, J. 1996. Stratification and power: structures of class, status, and command. Cambridge, Mass.: Polity Press.

SHILS, E. 1975. Centre and periphery: essays on macrosociology. Chicago: University of Chicago Press.

SOROKIN, P. 1964. Social and cultural mobility. New York: Free Press.

SØRENSEN, A. 1994. "Concluding commentary: class, status and power". In: GRUSKY, D. G. (ed.). Social stratification: class, gender and race in sociological perspective. Boulder: Westview Press.

TREIMAN, D. J. 1977. Occupational prestige in comparative perspective. New York: Academic Press.

TURNER, B. 1988. Status. Minneapolis: University of Minnesota Press.

WARNER, W. L.; MEEKER, M.; EELLS, K. 1960. Social class in America. New York: Harper and Row.

WEBER, M. 1978. Economy and society. Berkeley: University of California Press.

WEBSTER, M. A.; DRISKELL, J. E. 1978. "Status generalization: a review and some new data”. American Sociological Review, vol. 43, pp. 220-236.

WEGENER, B. 1992. "Concepts and measurement of prestige". Annual Review of Sociology, vol. 18, pp. 253-280. 


\section{STATUS EM SOCIEDADES PÓS-MODERNAS: A RENOVAÇÃO DE UM CONCEITO}

\section{MICHÈLE OLLIVIER}

Este artigo examina as várias formas com que o termo status tem sido definido na sociologia e identifica algumas das limitações dos conceitos clássicos para a compreensão da desigualdade nas sociedades contemporâneas. Defende que os conceitos neodurkheimianos baseiam-se em pressupostos insustentáveis a respeito do papel integrador do status 230 no contexto atual de relações sociais múltiplas, segmentadas e indiretas. Os conceitos neoweberianos, por sua vez, são úteis para se compreender como avaliações de superioridade são utilizadas por grupos como forma de legitimar a exclusão social, mas são menos úteis para compreender como avaliações coletivas de superioridade e inferioridade resultam na desigualdade entre indivíduos formalmente iguais em ambientes competitivos.

Palavras-chave: Teorias de status; Estratificação social; Prestígio; Sociedades contemporâneas.

\section{STATUS IN POSTMODERN SOCIETIES: THE RENEWAL OF A CONCEPT}

This paper examines the various ways in which status has been defined in sociology and identifies some of the limitations of classical concepts for understanding inequality in contemporary societies. It argues that neo-Durkheimian concepts of status rest on untenable assumptions concerning the integrative role of status in the current 
context of multiple, segmented, and indirect social relations. NeoWeberian concepts are useful for understanding how evaluations of superiority are used by groups to legitimate social exclusion but are less useful for understanding how collective evaluations of superiority and inferiority lead to inequality among formally equal individuals in competitive environments.

Keywords: Status theories; Social stratification; Prestige; Contemporaries societies. 